\title{
Amplification of laser excited capillary waves in a resonator
}

\author{
Al.A. Kolomenskii(1), E.C. Benck and H.A. Schuessler \\ Department of Physics, Texas A\&M University, College Station, TX 77843-4242, U.S.A.
}

\begin{abstract}
A modulated laser beam was used to generate capillary waves in a strongly absorbing liquid. The amplitude of the wave can be enhanced by placing the liquid into a resonant cylindrical container. At the proper modulation frequencies well pronounced resonances and standing waves were observed. The surface tension and viscosity of the liquid were measured.
\end{abstract}

\section{INTRODUCTION}

The study of a liquid surface is important for investigations of the heat and mass transfer through the surface, for observing molecular forces, and for obtaining the parameters of a liquid such as for instance surface tension and viscosity. A promising method for such investigations is the excitation of capillary waves by a modulated laser beam $[1,2]$. In the present paper we examine the generation of capillary waves inside a resonant mechanical cavity, and we make use of the amplification of the signal amplitude by the quality factor of the resonator.

\section{THEORY}

In the case of small dissipation the dispersion equation relating the eigenfrequency $\Omega_{\mathrm{n}}$ and the eigenvalue of the wave number $K_{n}$ for surface waves in a cylindrical container of radius $b$ and height of the liquid $h$ is given by

$$
\Omega_{\mathrm{n}}^{2}=\left(\mathrm{gK}_{\mathrm{n}}+\sigma \mathrm{K}_{\mathrm{n}}{ }^{3} / \rho\right) \tanh \left(\mathrm{K}_{\mathrm{n}} \mathrm{h}\right),
$$

where $g$ is the acceleration of gravity, and $\sigma$ is surface tension coefficient. The numbers $K_{n}$ are solutions of the equation $J_{1}\left(K_{n} b\right)=0$, where $K_{n}=m_{n} / b$, and $m_{n}$ are the zeros of the Bessel function $\mathrm{J}_{1}$. The complex amplitude of the excited capillary wave is proportional to the geometrical factor $F$ which describes the interference of the partial waves from different points of the distributed source. For a linear thermo-optical generation mechanism the factor 


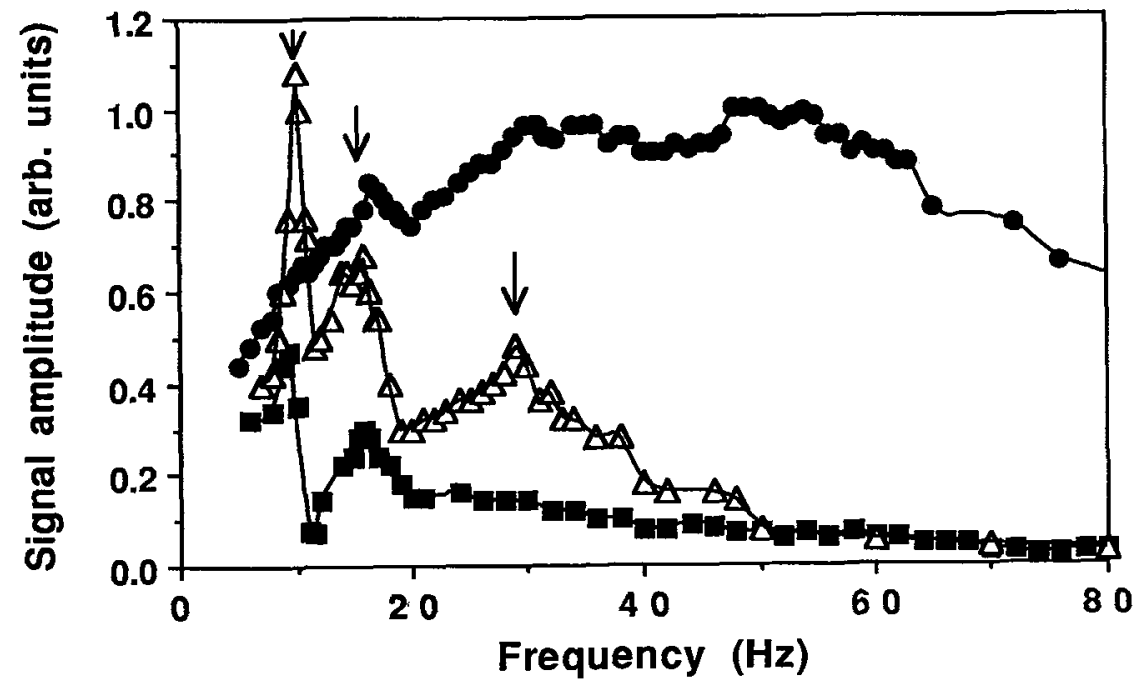

Fig. 1. Frequency dependence of the signal for different radii of the focused laser spot: $a=2.2 \mathrm{~mm}(\bullet), a=1.8 \mathrm{~mm}(\Delta), a=0.2 \mathrm{~mm}(\boldsymbol{\Pi})$. Parameters: observation distance $\mathrm{d}=0.4 \mathrm{~cm}$, laser power $150 \mathrm{~mW}$. The arrows indicate the first, second, and third cavity resonances.

$F$ is determined by the Fourier transform of the laser intensity distribution. In the case that the laser beam is centered in the middle of the resonator and that it has a Gaussian distribution this factor is $\mathrm{F}=\exp \left(-\mathrm{K}_{\mathrm{n}}^{2} a^{2} / 4\right)$. Here $a$ is the radius of a laser focus. In a resonator the amplification of the amplitude of a capillary wave is enhanced by the quality factor $\mathrm{Q}=\left(\Omega_{\mathrm{n}} \tau_{\mathrm{n}}\right)^{-1}$, where $\tau_{\mathrm{n}}=\left(\mathrm{g} / 2 \eta \mathrm{K}_{\mathrm{n}}{ }^{2}\right)$ is the damping time of the capillary wave. $\rho$ and $\eta$ are respectively the density and the viscosity of the liquid [3].

\section{EXPERIMENTAL APPARATUS}

Surface waves were excited using an Ar-ion laser operating at $\lambda=515 \mathrm{~nm}$. The intensity of the laser beam was modulated with frequencies ranging from $5 \mathrm{~Hz}$ to $2 \mathrm{kHz}$ employing a stabilized chopper. The laser beam was directed normally to the liquid surface of a dye solution and arranged to be at the center of a cylindrical container with a height $h=14 \mathrm{~mm}$ and a radius $b=13.5 \mathrm{~mm}$. The dye solution used was $1.25 \mathrm{~g} / \mathrm{L}$ of LDS 751 dissolved in ethylene glycol. The liquid filled the container up to the edge to avoid an influence of capillary effects from the wall of the container. The surface inclination angle of the liquid at the observation point of the capillary wave was measured with a probe He-Ne laser beam. Varying the modulation frequency we excited different modes of the resonator.

\section{RESULTS}

The frequency dependence of the capillary wave amplitude was observed for different values of the radii of the focused laser spot. Fig. 1 compiles the results. For a laser focus with $a=2.2 \mathrm{~mm}$ two resonances manifest themselves. For $a=1.8 \mathrm{~mm}$ three resonances are 
observable. The different resonances are located at $f_{1}=10 \mathrm{~Hz}, f_{2}=17 \mathrm{~Hz}$, and $f_{3}=30 \mathrm{~Hz}$. Decreasing the diameter of the focal spot increases the number $\mathrm{N}$ of the excited capillary wave modes of the resonator. This number is approximately determined by the condition $\mathrm{N}<\mathrm{b} / 2 \pi a$. The excitation and interference of a large number of modes leads to a reduction in contrast and even to the disappearance of the observable resonances when, as in our case, the quality factor is not very high. The reduction in amplitude with frequency occurs much faster in the case of the larger laser focal spots than in the tightly focused case and is due to the rapid decrease of the geometrical factor $F$. Comparatively the influence of damping on the amplitude is much smaller. An evaluation of the resonances yields the values for the viscosity to be $\eta=0.68 \mathrm{~g} / \mathrm{cm} \mathrm{sec}$ and the surface tension to be $\sigma=54 \mathrm{erg} / \mathrm{cm}^{2}$. These values agree with those measured using conventional techniques.

This work is supported by a Teledyne Research Assistance Grant, DOE, and the Center for Energy and Mineral Resources of Texas A\&M University.

\section{References}

[1] J. Hartikainen, J. Jaarinen, and M. Luukkala, Can. J. Phys. 64 (1986) 1341.

[2] B. M. Grigorova, S. F. Rastopov, A. T. Sukhodolskii, Zh. Tekh. Fiz. 60 (1990) 167.

[3] Al. A. Kolomenskii, Sov. J. Quantum Electronics 19 (1989) 365. 